\title{
Limit Laws for Random Spatial Graphical Models
}

\author{
Animashree Anandkumar*, Joseph Yukich ${ }^{\dagger}$, and Alan Willsky* \\ *EECS Dept., MIT, Cambridge, MA 02139, USA. Email: \{animakum,willsky\}@ mit.edu \\ ${ }^{\dagger}$ Dept. of Math., Lehigh University, Bethlehem, Pa. 18015, USA. Email: joseph.yukich@lehigh.edu
}

\begin{abstract}
We consider spatial graphical models on random Euclidean points, applicable for data in sensor and social networks. We establish limit laws for general functions of the graphical model such as the mean value, the entropy rate etc. as the number of nodes goes to infinity under certain conditions. These conditions require the corresponding Gibbs measure to be spatially mixing and for the random graph of the model to satisfy a certain localization property known as stabilization. Graphs such the $k$ nearest neighbor graph and the geometric disc graph belong to the class of stabilizing graphs. Intuitively, these conditions require the data at each node not to have strong dependence on data and positions of nodes far away. Finally, it is shown that spatial mixing of the Gibbs measure on a random graph holds when a suitably defined degree-dependent (but otherwise independent) node percolation does not have a giant component.
\end{abstract}

Index Terms-Spatial graphical models, random Euclidean graphs, phase transition, spatial mixing, stabilization.

\section{INTRODUCTION}

Graphical models incorporate complex relationships among random variables on a dependency graph, where the nodes in the graph represent the variables under consideration [1]. Roughly, neighboring nodes in the dependency graph are strongly related to one another, while nodes that are well separated on the dependency graph are weakly related.

A key question for large-scale graphical models is the notion of correlation decay: does the correlation between nodes decay with their graph distance? This is most extensively studied in the Ising model on a regular lattice grid [2], where it captures the phase transition between the gaseous and liquid states: in the gaseous state, there are only weak forces among the nodes (molecules) and correlation decay occurs, while in the liquid state, the nodes have strong attractive forces and there is no correlation decay. Establishing correlation decay in large-scale graphical models has many implications, such as efficient local computation of various functions of the graphical model [3][5]. Extensive results on correlation decay are available for various models when the dependency graph is either a grid graph or a tree [2].

In this paper, we provide sufficient conditions for correlation decay in random spatial graphical models. We consider graphical model over $n$ nodes with correlated data placed randomly in growing regions on $\mathbb{R}^{d}$ and study the scaling laws for certain functions of the data, as we let $n \rightarrow \infty$. This model is applicable in many scenarios such as a sensor

The first and the third authors are supported by AFOSR MURI Grants FA9550-061-0324 and FA9559-08-1-0180. The second author is supported in part by NSF grant DMS-0805570. network deployed for environmental monitoring applications or a social network among people in a geographic region, where opinions are formed and propagated [6].

We limit our focus to a general class of proximity-based Euclidean random graphs satisfying a localization property called stabilization, e.g., the $k$ nearest neighbor graph and the geometric disc graph. Scaling laws for such graphs have been characterized in [7], [8]. However, the results in [7], [8] only hold when the measurements at the nodes are i.i.d. and independent of the graph. In contrast, we consider nodes having measurements whose joint probability distribution is a Gibbs measure on a random Euclidean graph.

Our contributions are three fold. First, we establish conditions for existence of limits and derive the limits for a general class of functions of spatial graphical models on random Euclidean graphs. Second, we relate these conditions to notions of correlation decay of the Gibbs measure ${ }^{1}$ (spatial mixing) [9] and stabilization property of the graph of the Gibbs measure [7], [8]. Third, we study specific graphs and models where these conditions hold. We defer the detailed proofs to the full version of this paper.

\section{SyStem MOdEL AND FORMULATION}

In this section, we introduce the model for node placement and then describe the graphical model for the node measurements given the node locations. Finally, we discuss some functions of the graphical model which are of interest.

\section{Notation}

For any finite set $\mathbf{C}$, let $|\mathbf{C}|$ denote its cardinality.Let $\mathcal{G}=\left(\mathbf{V}, E_{\mathcal{G}}\right)$ denote an undirected graph over $\mathbf{V}$. Let $E(i ; \mathcal{G})$ denote edges incident to node $i, \operatorname{deg}(i)$ its degree, $\mathcal{N}(i ; \mathcal{G})$ its (immediate) neighbors and let $\mathcal{N}^{s}(i ; \mathcal{G})$ its $s$-neighborhood, i.e., the set of nodes within $s$ hops from $i$. For any two nodes $V_{i}, V_{j} \in \mathbb{R}^{d}$, let $\operatorname{dist}_{\text {euc }}\left(V_{i}, V_{j}\right)$ denote the Euclidean distance and let $\operatorname{dist}_{\mathcal{G}}\left(V_{i}, V_{j}\right)$ denote the graph distance (minimum number of hops between $V_{i}$ and $V_{j}$ on graph $\mathcal{G}$ ).

Let $\mathbf{0} \in \mathbb{R}^{d}$ denote the origin and $B_{r}(V) \subset \mathbb{R}^{d}$ denote the ball of radius $r$ centered at location $V \in \mathbb{R}^{d}$. Let $\mathcal{P}_{\tau}$ denote the homogeneous Poisson distribution on $\mathbb{R}^{d}$ with intensity $\tau$ and let $\mathcal{P}_{\tau, \mathbf{0}}:=\mathcal{P}_{\tau} \cup\{\mathbf{0}\}$. Let $\mathrm{d}_{\mathrm{TV}}(P, Q)$ denote the total variation distance between two measures $P$ and $Q$, given by

$$
\mathrm{d}_{\mathrm{TV}}(P, Q):=\sup _{\mathcal{K}}|P[\mathcal{K}]-Q[\mathcal{K}]|,
$$

\footnotetext{
${ }^{1}$ We use the terms graphical model and Gibbs measure interchangeably.
} 
over all Borel sets $\mathcal{K}$ in $\mathbb{R}^{d}$. When $X$ and $Y$ are variables defined on a common measure $\nu$, with abuse of notation,

$$
\mathrm{d}_{\mathrm{TV}}(X, Y):=\sup _{\mathcal{K}}|\nu[X \in \mathcal{K}]-\nu[Y \in \mathcal{K}]| .
$$

\section{A. Random Node Locations}

We consider $n$ nodes randomly located in growing regions on $\mathbb{R}^{d}$ taking correlated measurements. Let $V_{i} \in \mathbb{R}^{d}$ denote the location of node $i$. We assume that all the locations $V_{i}$ are generated in an i.i.d. manner as follows: let $X_{1}, X_{2}, \ldots, X_{n} \stackrel{i . i . d .}{\sim} f$, where $f$ is any pdf with support ${ }^{2}$ on the unit ball $B_{1}(\mathbf{0})$ with,

$$
0 \leq f_{\min } \leq f(x) \leq f_{\max } \leq \infty, \quad x \in B_{1}(\mathbf{0})
$$

The node locations are the scaled versions of $X_{i}$, i.e., $V_{i}=$ $\sqrt[d]{\frac{n}{\lambda}} X_{i}$. Hence, $n$ nodes are located randomly in volume $\frac{n}{\lambda}$.

We let $n \rightarrow \infty$, while keeping the density $\lambda$ and the dimension of the Euclidean space $d$ fixed. For the special case of uniform node placement $(f \equiv 1)$, as $n \rightarrow \infty$, the set of node locations behave locally ${ }^{3}$ like a homogeneous Poisson process $\mathcal{P}_{\lambda}$ and for general node placements $f$, they behave locally like a Cox process [8, Lemma 3.1].

\section{B. Gibbs Model for Node Measurements}

The measurement $Y_{i}$ at each node $i$ is discrete with a finite sample space $\mathcal{Y}$ with cardinality $|\mathcal{Y}|$. In order to explicitly denote the measurements of nodes at locations $\mathbf{V}_{n}$, we sometimes use the alternative notation $\mathbf{Y}_{\mathbf{V}_{n}}$, in place of $\mathbf{Y}_{n}$. We assume that $\mathbb{P}\left[\mathbf{Y}_{n} \mid \mathbf{V}_{n}=\mathbf{v}_{n}\right]$ follows a pairwise Gibbs measure.

Definition 1 (Gibbs Process): A pairwise Gibbs measure is

$$
\mathbb{P}\left[\mathbf{Y}_{n} \mid \mathbf{V}_{n}=\mathbf{v}_{n}\right]=\frac{1}{Z_{\mathbf{v}_{n}}} e^{\beta \sum_{(i, j) \in \mathcal{G}\left(\mathbf{v}_{n}\right)} \Psi_{v_{i}, v_{j}}\left(Y_{i}, Y_{j}\right)+\gamma \sum_{i=1}^{n} \Phi_{v_{i}}\left(Y_{i}\right)},
$$

where $\mathcal{G}\left(\mathbf{v}_{n}\right)$ is a graph defined on the Euclidean points, usually known as the dependency graph. In (1), $0<Z_{\mathbf{v}_{n}}<\infty$ is the normalizing constant, known as the partition function. The functions $\Psi_{i, j}$ are known as the edge potentials and $\Phi_{i}$ as the node potentials.

For example, in the Ising model, $\Psi\left(Y_{i}, Y_{j}\right)=Y_{i} Y_{j}$ and $\Phi\left(Y_{i}\right)=Y_{i}$. In this paper, we consider the dependency graph for node measurements $\mathcal{G}\left(\mathbf{v}_{n}\right)$ modeled by a random Euclidean graph, where the randomness arises due to the random locations of the nodes and the graph is based on the Euclidean distances between the node pairs. Henceforth, we use the notation $\mathbb{P}_{\mathcal{G}\left(\mathbf{v}_{n}\right)}\left[\mathbf{Y}_{n} \mid \mathbf{V}_{n}=\mathbf{v}_{n}\right]$ to explicitly denote the Gibbs process with $\mathcal{G}\left(\mathbf{v}_{n}\right)$ as the dependency graph.

The Gibbs process $\mathbb{P}\left[\mathbf{Y}_{n} \mid \mathbf{V}_{n}=\mathbf{v}_{n}\right]$ can alternatively be specified through a Markovian property on the graph $\mathcal{G}\left(\mathbf{v}_{n}\right)$ for distributions satisfying the positivity condition: $\mathbb{P}\left[\mathbf{y}_{n} \mid \mathbf{V}_{n}=\right.$ $\left.\mathbf{v}_{n}\right]>0$ for all possible values $\mathbf{y}_{n} \in \mathcal{Y}^{n}$, i.e., there are no hardcore constraints. In this case,

$$
Y_{i} \Perp \mathbf{Y}_{\mathbf{V}_{n} \backslash \mathcal{N}(i ; \mathcal{G})} \mid\left\{\mathbf{Y}_{\mathcal{N}(i ; \mathcal{G})}=\mathbf{y}_{\mathcal{N}(i ; \mathcal{G})}, \mathbf{V}_{n}=\mathbf{v}_{n}\right\}
$$

${ }^{2}$ Our results hold in the general case when $f$ has some compact support. ${ }^{3}$ Specifically, $\lim _{n \rightarrow \infty} \mathrm{d}_{\mathrm{TV}}\left(\mathcal{P}_{\lambda} \cap B_{r}(\mathbf{0}), \mathbf{V}_{n} \cap B_{r}(\mathbf{0})\right)=0$ for any finite $r>0$. where $\Perp$ denotes conditional independence. Hence, the measurement at a node is conditionally independent of the rest of the network, given the node locations and the measurements at its neighbors in the dependency graph, and is formally known as a Markov random field [10].

To obtain scaling behavior as $n \rightarrow \infty$, we assume a homogeneous translation-invariant model,

$$
\mathbb{P}\left[\mathbf{Y}_{n} \mid \mathbf{V}_{n}=\mathbf{v}_{n}\right]=\mathbb{P}\left[\mathbf{Y}_{n} \mid \mathbf{V}_{n}=\mathbf{v}_{n}+v\right], \quad \forall v \in \mathbb{R}^{d} .
$$

This implies that the node potentials are identical $\left(\Phi_{v_{i}} \equiv \Phi\right)$ for all $i=1, \ldots, n$, and that the edge potentials to be translation invariant. Additionally, $\mathcal{G}\left(\mathbf{v}_{n}\right)$ is also translation invariant. We limit ourselves to stabilizing graphs, defined later in Definition 4, such as the $k$ nearest neighbor graph and the geometric disc graph.

\section{Functions of Interest}

Perhaps, the simplest functional is the limiting mean value

$$
\bar{Y}_{\beta, \gamma, \lambda}:=\lim _{n \rightarrow \infty} \frac{1}{n} \sum_{i=1}^{n} \mathbb{E}\left[Y_{i}\right] .
$$

The partition function $Z$ and its limit are of interest in many applications [1],

$$
\Upsilon_{\beta, \gamma, \lambda}:=\lim _{n \rightarrow \infty} \frac{1}{n} \log Z_{\mathbf{V}_{n}, \beta, \gamma}, \quad\left(\frac{n}{\lambda}\right)^{-\frac{1}{d}} \mathbf{V}_{n} \stackrel{\text { i.i.d. }}{\sim} f,
$$

The above constant $\Upsilon$ (or sometimes its scaled version) is known as the pressure or specific Gibbs free energy [2, Def. 15.36]. The derivative of the pressure with respect to the parameters $\beta$ (usually known as inverse temperature) yields

$$
\frac{\partial}{\partial \beta} \Upsilon_{\beta, \gamma, \lambda}=-\lim _{n \rightarrow \infty} \frac{1}{n} \sum_{(i, j) \in \mathcal{G}\left(\mathbf{V}_{n}\right)} \mathbb{E}\left[\Psi\left(Y_{i}, Y_{j}\right)\right]
$$

with $\left(\frac{\lambda}{n}\right)^{\frac{1}{d}} \mathbf{V}_{n} \stackrel{i . i . d .}{\sim} f$. Hence, the existence of the limit in a certain regime of $\beta$ implies the existence of limit in (4).

Another important quantity is the specific entropy,

$$
h_{\beta, \gamma, \lambda}:=\lim _{n \rightarrow \infty} \frac{H\left(\mathbf{Y}_{n} \mid \mathbf{V}_{n}\right)}{n}=\mathbb{E}\left[\Upsilon_{\beta, \gamma, \lambda}+\Gamma_{\beta, \gamma, \lambda}\right],
$$

where $\Gamma_{\beta, \gamma, \lambda}$ is the specific Gibbs internal energy, given by

$$
\Gamma_{\beta, \gamma, \lambda}:=-\lim _{n \rightarrow \infty} \frac{1}{n}\left[\beta \sum_{(i, j) \in \mathcal{G}\left(\mathbf{V}_{n}\right)} \Psi\left(Y_{V_{i}}, Y_{V_{j}}\right)+\gamma \sum_{i=1}^{n} \Phi\left(Y_{V_{i}}\right)\right] .
$$

Our goal is to find the regimes where limits in (3), (5), and (6) exist by casting them as functionals over a marked point process. Let $M_{i}:=\left(V_{i}, Y_{i}\right)$ denote the tuple for node $i$, consisting of its location $V_{i} \in \mathbb{R}^{d}$ and its measurement value $Y_{i}$. Let $\mathbf{M}_{n}:=\left\{M_{1}, M_{2}, \ldots, M_{n}\right\}$ denote the set of node location-measurement pairs of all the $n$ nodes. We also use the alternative notation $\mathbf{M}\left(\mathbf{V}_{n}\right)$ to denote the set of node-location pairs $\mathbf{M}_{n}$ with locations fixed at $\mathbf{V}_{n}$.

For each marked point $M_{i} \in \mathbf{M}_{n}$, let $\xi\left(M_{i} ; \mathbf{M}_{n}\right)$ denote a translation-invariant functional which specifies the relationship 
of the marked point $M_{i}$ to the entire set $\mathbf{M}_{n}$. We focus on functionals of the form

$$
\xi\left(M_{i} ; \mathbf{M}\left(\mathbf{V}_{n}\right)\right)=\xi\left(M_{i} ; \mathbf{M}\left(\mathcal{N}\left(i ; \mathcal{G}\left(\mathbf{V}_{n}\right)\right)\right)\right),
$$

consisting only of relationships between a node and its neighbors and holds for the summands in (3), (5), and (6).

\section{LIMIT LAWS FOR GIBBS FUNCTIONALS}

We prove the existence and find the limit

$$
\lim _{n \rightarrow \infty} \frac{1}{n} \sum_{i=1}^{n} \mathbb{E}\left[\xi\left(M_{i} ; \mathbf{M}_{n}\right)\right]
$$

For the existence of the above limit, we expect the value of functional $\xi$ at each node not to have strong dependence on nodes "far away" from it. We formalize this notion through the concept of weak stabilization.

\section{A. Weak Stabilization}

For any marked point set $\mathbf{M}=\left(\mathbf{V}, \mathbf{Y}_{\mathbf{V}}\right)$ where the point set $\mathbf{V} \in \mathbb{R}^{d}$ is locally finite, let

$$
\begin{aligned}
& \bar{\xi}\left(\mathbf{M}_{\mathbf{V}}, r\right):=\underset{\mathcal{A} \subset \mathbb{R}^{d} \backslash B_{r}(\mathbf{0})}{\operatorname{ess} \sup _{(10}} \xi\left(M_{\mathbf{0}}, \mathbf{M}\left(\left(\mathbf{V} \cap B_{r}(\mathbf{0})\right) \cup \mathcal{A}\right)\right), \\
& \underline{\xi}\left(\mathbf{M}_{\mathbf{V}}, r\right):=\underset{\mathcal{A} \subset \mathbb{R}^{d} \backslash B_{r}(\mathbf{0})}{\operatorname{essinf}} \xi\left(M_{\mathbf{0}}, \mathbf{M}\left(\left(\mathbf{V} \cap B_{r}(\mathbf{0})\right) \cup \mathcal{A}\right)\right),
\end{aligned}
$$

where ess sup and essinf denote essential supremum and infimum, (i.e., almost everywhere).

Definition 2 (Weakly Stabilizing Functional): A functional $\xi$ on marked points is said to be weakly stabilizing on a marked point set $\mathbf{M}(\mathbf{V})$ where $\mathbf{V} \in \mathbb{R}^{d}$ is locally finite if

$$
\lim _{r \rightarrow \infty} \mathrm{d}_{\mathrm{TV}}\left(\bar{\xi}\left(\mathbf{M}_{\mathbf{V}}, r\right), \underline{\xi}\left(\mathbf{M}_{\mathbf{V}}, r\right)\right)=0 .
$$

The above condition states that the effect of faraway nodes (their measurements and locations) on the value of functional $\xi$ decays with distance. We require weak stabilization of the functional $\xi$ on homogeneous Poisson processes $\mathcal{P}_{\tau}$ for some range of intensities $\tau$.

\section{B. Law of Large Numbers}

We now show that the limit in (8) exists when weak stabilization in (9) is satisfied on homogeneous Poisson processes. We also assume bounded moments condition on $\xi$ [8].

$$
\sup _{n \in \mathbb{N}} \mathbb{E}\left|\xi\left(M_{1}, \mathbf{M}_{n}\right)\right|^{p}<\infty, \quad \text { for some } p>1 \text {. }
$$

Theorem 1 (LLN for Weakly Stabilizing Functionals):

For a functional $\xi$ weakly stabilizing according to (9) on homogeneous Poisson process $\mathcal{P}_{\tau}$, for all $\tau \in\left[\lambda f_{\min }, \lambda f_{\max }\right]$, where $f$ is the node placement pdf with bounds $f_{\min }$ and $f_{\max }$ and $\xi$ has bounded moments in (10), we have

$$
\begin{aligned}
& \frac{1}{n} \sum_{i=1}^{n} \mathbb{E}\left[\xi\left(M_{i} ; \mathbf{M}\left(\mathbf{V}_{n}\right)\right)\right] \stackrel{n \rightarrow \infty}{\longrightarrow} \\
& \int_{B_{1}(\mathbf{0})} \mathbb{E}\left[\xi\left(M_{\mathbf{0}} ; \mathbf{M}\left(\mathcal{P}_{\lambda f(y)}\right)\right)\right] f(y) d y<\infty .
\end{aligned}
$$

Remark 1: For uniform node placement pdf $(f \equiv 1),(11)$ simplifies to

$$
\frac{1}{n} \sum_{i=1}^{n} \mathbb{E}\left[\xi\left(M_{i} ; \mathbf{M}\left(\mathbf{V}_{n}\right)\right)\right] \stackrel{n \rightarrow \infty}{\longrightarrow} \mathbb{E}\left[\xi\left(M_{\mathbf{0}} ; \mathbf{M}\left(\mathcal{P}_{\lambda}\right)\right)\right], \quad f \equiv 1 .
$$

Hence, the limit of expected value of functions $\xi$ at the nodes of a graphical model exists under weak stabilization. Moreover, the limit is given by a local quantity, i.e., the expectation of the function at the origin over a homogeneous Poisson process, in case of uniform node placement. For nonuniform node placement, the intensity of the corresponding Poisson process depends on the node placement pdf $f$.

\section{Sufficient Conditions for Weak Stabilization}

The existence of the limit in (11) thus requires stabilization, which in turn imposes constraints on dependency graph $\mathcal{G}$ and on the model parameters in (1). In general, it is not tractable to establish the exact stabilization regime in (9), and we instead establish some sufficient conditions in terms of spatial mixing of the Gibbs measure.

\section{A. Spatial Mixing}

The notion of spatial mixing is used here to limit the influence of a node's measurement on the conditional distribution of measurements at other nodes.

Definition 3 (Almost-Sure (Weak) Spatial Mixing [9]): A Gibbs process $\mathbb{P}\left[\mathbf{Y}_{\mathbf{V}}\right]$ on a locally finite node set $\mathbf{V}$ exhibits weak spatial mixing a.s., when for measurement $Y_{v}$ at every point $v \in \mathbf{V}$ and any node subset $\mathcal{V} \subset \mathbf{V}$, we have a.s.,

$$
\mathrm{d}_{\mathrm{TV}}\left(\mathbb{P}\left[Y_{v} \mid \mathbf{Y}_{\mathcal{V}}=\mathbf{y}_{\mathcal{V}}\right], \mathbb{P}\left[Y_{v} \mid \mathbf{Y}_{\mathcal{V}}=\mathbf{z}_{\mathcal{V}}\right]\right) \leq \delta\left(\operatorname{distg}_{\mathcal{G}}(v, \mathcal{V})\right)
$$

for any two feasible configurations $\mathbf{y} \mathcal{V}, \mathbf{z}_{\mathcal{V}} \in \mathcal{Y}^{|\mathcal{V}|}$, such that $\lim _{s \rightarrow \infty} \delta(s)=0$.

$s \rightarrow \infty$ Hence, spatial mixing implies correlation decay over long distances since the influence of a node on another node decreases with their graph distance. More specifically, (12) implies the asymptotic independence between the measurement at a node and an expanding boundary.

Comparing the notion of spatial mixing in (12) with that of stabilization in (9), we note two important differences. First, the spatial-mixing conditions are described in terms graph distance distg while stabilization is in terms of Euclidean distance dist $_{\text {euc }}$. Second, the spatial-mixing condition only deals with the measurements of far away nodes while the stabilization condition deals with both the measurements and locations of far away nodes. The locations of far away nodes also influence the measurement value at the origin, indirectly through the configuration of the random graph $\mathcal{G}$. Hence, spatial mixing, by itself, is not sufficient to guarantee stabilization for graphical models on randomly placed nodes.

\section{B. Stabilizing Graphs}

As stated before, we limit ourselves to dependency graphs of the graphical model in (1) which are strongly stabilizing. We now formally define this property. 
Definition 4 (Strongly Stabilizing Graph [8]): A translation-invariant graph $\mathcal{G}(\mathbf{V})$ on locally-finite points $\mathbf{V}$ with $\mathbf{0} \in \mathbf{V}$ is strongly stabilizing with an a.s. finite (random) radius of stabilization $R<\infty$ if for all locally finite sets $\mathcal{A} \subset \mathbb{R}^{d} \backslash B_{R}(\mathbf{0})$,

$$
E\left(\mathbf{0} ; \mathcal{G}\left(\left(\mathbf{V} \cap B_{R}(\mathbf{0})\right) \cup \mathcal{A}\right)\right)=E\left(\mathbf{0}, \mathcal{G}\left(\mathbf{V} \cap B_{R}(\mathbf{0})\right) .\right.
$$

The radius of stabilization $R$ is defined to be the smallest integer for which (13) holds.

Hence, a strongly ${ }^{4}$ stabilizing graph has localized edges: the edges of a node in the graph are immune to changes made outside the ball centered at that node with a finite radius $R<\infty$. A wide range of proximity graphs satisfy the strong stabilization property in (13) such as the $k$ nearest-neighbor graph, the geometric disc graph, minimum spanning tree, and so on; see [7] for an extensive list of strongly stabilizing graphs.

The strong stabilization condition in (13) rules out any longrange edges, and hence, there cannot be short graph distances between spatially well-separated nodes. This is useful in relating the spatial mixing condition in (12) to the stabilization condition in (9) since here, the graph distances grow with Euclidean distances.

We now prove another property of a strongly stabilizing graph which characterizes the effect on the extended neighborhood of the origin $\mathcal{N}^{s}(\mathbf{0})$ for some $s$, due to changes made faraway from the origin. To this end, define for $R_{s}, r>0$,

$$
M(r):=\inf \left[s: R_{s}>r, s \in \mathbb{N}\right] .
$$

Lemma 1: A strongly stabilizing graph $\mathcal{G}$ on the Poisson process $\mathcal{P}_{\tau, \mathbf{0}}$ is also strongly stabilizing with respect to its $s$-hop neighborhood $\mathcal{N}^{s}(\mathbf{0})$, for any finite $s \in \mathbb{N}$,

$\mathcal{N}^{s}\left(\mathbf{0}, \mathcal{G}\left(\mathcal{P}_{\tau, \mathbf{0}} \cap B_{R_{s}}(\mathbf{0})\right)\right)=\mathcal{N}^{s}\left(\mathbf{0}, \mathcal{G}\left(\left(\mathcal{P}_{\tau, \mathbf{0}} \cap B_{R_{s}}(\mathbf{0})\right) \cup \mathcal{A}\right)\right)$,

for all locally-finite $\mathcal{A} \subset \mathbb{R}^{d} \backslash B_{R_{s}}(\mathbf{0})$, where $R_{s}\left(\mathcal{P}_{\tau}\right)<\infty$ (a.s.) is the $s$-hop stabilization radius. For $M(r)$ defined in (14), under Poisson process $\mathcal{P}_{\tau, \mathbf{0}}$,

$$
\lim _{r \rightarrow \infty} s^{*}(r)=\infty, \quad \text { a.s. }
$$

Hence, a strongly stabilizing graph is also stabilizing with respect to its $s$-hop neighborhood, for any finite $s \in \mathbb{N}$, and has radius of stabilization $R_{s}\left(\mathcal{P}_{\tau, 0}\right)$. This implies that changing locations of nodes and/or adding deleting nodes outside the ball $B_{r}(\mathbf{0})$ can only change the graph beyond $M(r)$ hops from the origin and the $(M(r)-1)^{+}$neighborhood of the origin is unchanged. Hence, we refer to $M(r)$ as the maximum $r$ stable neighborhood of the origin on a stabilizing graph over a Poisson point process. See Fig.1 for a pictorial representation. We will see that the above result is crucial in relating spatial mixing with stabilization.

\section{Sufficient Conditions for Weak Stabilization}

We now show that spatial mixing in (12) and stabilizing graph in (13) imply weak stabilization in (9).

${ }^{4}$ In order to distinguish from our weak stabilization definition in (9), we use the term strongly stabilizing in (13)

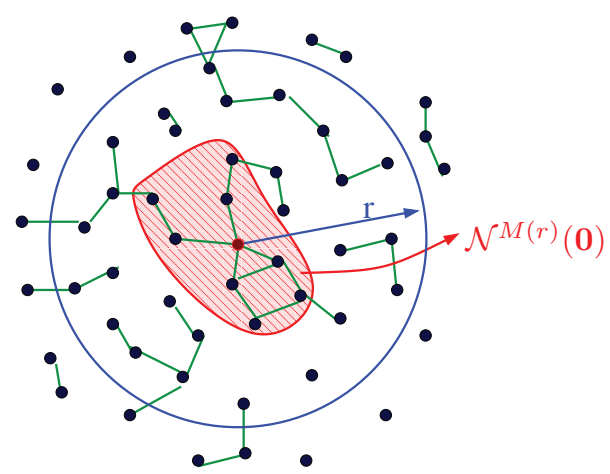

Fig. 1. Pictorial representation of maximum $r$-stable neighborhood $M(r)$. Changing the configuration of nodes beyond a distance $r$ affects only nodes beyond graph distance of $M(r)$. See (14) for definition of $M(r)$.

Theorem 2 (Weak Stabilization via Spatial Mixing): If a Gibbs process defined on a homogeneous Poisson process $\mathcal{P}_{\tau}$ is a.s. weak spatial mixing as in (12), and the dependency graph $\mathcal{G}$ of the Gibbs process is strongly stabilizing as in (13) on $\mathcal{P}_{\tau}$, then the functional of the form (7) weakly stabilizes according to (9) on $\mathcal{P}_{\tau}$.

Proof: Under spatial mixing in (12), we can consider a boundary $\mathcal{V}$ of nodes which are $M(r)$ hops away from the origin and by (15), since the maximum $r$-stable neighborhood $M(r)$ grows with $r$, the influence decays as $r$ grows and stabilization holds.

Hence, spatial mixing implies weak stabilization if the graph of the Gibbs process is strongly stabilizing. Recall that weak stabilization in turn implies existence of limits for general Gibbs functionals in Theorem 1. There are mainly two methods to establish regimes of spatial mixing and we discuss them now.

\section{Stabilization in Specific GRaphs}

\section{A. Stabilization Under Finite Maximum Degree}

We first consider spatial mixing in graphs which have a finite maximum degree $\Delta<\infty$ and are also strongly stabilizing. For instance, the $k$-nearest neighbor graph has $\Delta=\left(c_{d}+1\right) k$, where $c_{d}$ is a constant called the kissing number [11, Cor. 3.2.3]. Intuitively, when the Gibbs potentials are weak and there are no high degree nodes in the graph, the influence of far away nodes is limited and spatial mixing holds.

Formally, the (Dobrushin) influence coefficient of $\mathrm{t}$ a node $j$ on node $i$ in a node set $\mathbf{V}$ is [9], [12],

$$
\begin{aligned}
C_{i, j} & :=\max _{\substack{\mathbf{y}, \mathbf{z} \in \mathcal{Y}^{|\mathbf{V}|-1} \\
y(k)=z(k), \forall k \neq j}} \mathrm{~d}_{\mathrm{TV}}\left(\mathbb{P}\left[Y_{i} \mid \mathbf{Y}_{\mathbf{V} \backslash i}=\mathbf{y}\right], \mathbb{P}\left[Y_{i} \mid \mathbf{Y}_{\mathbf{V} \backslash i}=\mathbf{z}\right]\right) . \\
&
\end{aligned}
$$

Note that from Markovian property in (2), $C_{i, j}=0$ if $j \notin$ $\mathcal{N}(i ; \mathcal{G})$. The maximum influence on any node is given by $\alpha:=\max _{i \in \mathbf{V}} \sum_{j \in \mathbf{V}} C_{i, j}$. We have Dobrushin's condition ${ }^{5}$

$$
\alpha<1 \Rightarrow \text { Spatial Mixing. }
$$

\footnotetext{
${ }^{5}$ There are a general set of sufficient conditions for spatial mixing in [12].
} 
If we consider a specific model in (1) such as the Ising model, then the above condition translates to a regime of inverse temperate $\beta<\beta_{c}(\Delta)$, for some critical value $\beta_{c}(\Delta)$ which depends on the maximum degree $\Delta$.

For graphs with no finite degree bound, the condition in (17) holds only under the trivial case of independent data at nodes as $n \rightarrow \infty$ and hence, we consider an alternative approach.

\section{B. Stabilization Under Growing Maximum Degree}

In this section, we focus on the geometric disc graph denoted by $\mathcal{G}\left(\mathbf{V}_{n} ; r_{n}\right)$. We set the threshold radius $r_{n}=1$ without loss of generality and vary intensity $\lambda$ instead. In this regime, the maximum degree in $\mathcal{G}\left(\mathbf{V}_{n} ; 1\right)$ is unbounded and grows as $\Theta\left(\frac{\log n}{\log \log n}\right)$ [13, Thm. 6.10].

We first consider the geometric disc graph or continuum percolation $\mathcal{G}\left(\mathcal{P}_{\lambda} ; 1\right)$ in the subcritical regime $\left(\lambda<\lambda_{c}(d)\right)$, where $\lambda_{c}(d)$ is the critical intensity [13, (9.12)]. Here, there is no giant component a.s. and it is easy to see that the effect of far away nodes does not propagate since the components are small. (Recall that measurements of nodes in different components are independent). We formally state that weak stabilization holds in this case.

Proposition 1 (Weak Stabilization in Subcritical Regime): Functionals $\xi$ of the form (7) are stabilizing on continuum percolation $\mathcal{G}\left(\mathcal{P}_{\lambda, \mathbf{0}}, 1\right)$ in the subcritical regime $\left(\lambda<\lambda_{c}(d)\right)$.

We now extend the above result to the super-critical regime $\left(\lambda>\lambda_{c}(d)\right)$ where there is a giant component, yet, spatial mixing occurs under certain conditions. We establish spatial mixing in this regime through disagreement percolation [14], [15], where a sufficient condition for spatial mixing is obtained through absence of giant component in an independent Bernoulli node percolation process on the graph, where the probability of picking a node is given by

$$
\rho(i):=\max _{\mathbf{y}, \mathbf{z} \in \mathcal{Y}|\mathbf{V}|-1} \operatorname{d}_{\mathbf{T V}}\left(\mathbb{P}\left[Y_{i} \mid \mathbf{Y}_{\mathbf{V} \backslash i}=\mathbf{y}\right], \mathbb{P}\left[Y_{i} \mid \mathbf{Y}_{\mathbf{V} \backslash i}=\mathbf{z}\right]\right) .
$$

We formally state this result below. Denote independent Bernoulli node percolation on $\mathcal{G}\left(\mathcal{P}_{\lambda} ; 1\right)$ as $\mathcal{G}\left(\mathcal{P}_{\lambda} ; 1, \rho(\cdot)\right)$ where $\rho(i)$ is the probability of selecting node $i$.

Lemma 2 (Stabilization Regime Through Percolation): If $\mathcal{G}\left(\mathcal{P}_{\lambda f_{\max }} ; 1, \rho(\cdot)\right)$ does not percolate, i.e., does not contain a giant component a.s. then the functional $\xi$ of the form in (7) is weakly stabilizing according to (9) and hence, we have existence of limits in Theorem 1.

To obtain results for Bernoulli percolation on the geometric disc graph, note the probabilities $\rho(\cdot)$ in (18) are not uniform and hence, the simple thinning results do not hold. In the special case of Ising model, it can be shown that $\rho(\cdot)$ is a non-decreasing function in the degree of the node. In this case, the results of [16] are applicable, where a sufficient condition for non-percolation of a degree-dependent percolation function is derived as an infinite series. Thus, the percolation-based approach provides non-trivial regimes for spatial mixing on the geometric disc graph while the Dobrushin condition in (17) fails due to the growing maximum degree.

\section{CONCLUSION}

We considered graphical models on random Euclidean points and derived limit laws for various functions, as the number of nodes goes to infinity. We derived sufficient conditions for existence of limits, by controlling the influence of the data and locations of faraway nodes. In future, we plan to investigate necessary conditions for existence of limits, as well as provide lower and upper bounds on functions, when the limits do not exist.

\section{Acknowledgements}

The authors thank Prof. D. Gamarnik and Prof. D. Shah at MIT for extensive discussions.

\section{REFERENCES}

[1] M. Wainwright and M. Jordan, "Graphical Models, Exponential Families, and Variational Inference," Foundations and Trends in Machine Learning, vol. 1, no. 1-2, pp. 1-305, 2008.

[2] H. Georgii, Gibbs Measures and Phase Transitions. Walter de Gruyter, 1988.

[3] S. Tatikonda and M. Jordan, "Loopy Belief Propagation and Gibbs Measures," in Uncertainty in Artificial Intelligence, vol. 18, 2002, pp. 493-500.

[4] T. Hayes, "A Simple Condition Implying Rapid Mixing of SingleSite Dynamics on Spin Systems," in 47th Annual IEEE Symposium on Foundations of Computer Science, 2006, pp. 39-46.

[5] D. Gamarnik and D. Katz, "Sequential Cavity Method for Computing Limits of the Log-Partition Function for Lattice Models," in SODA '09: Proc. of ACM-SIAM Symp. on Discrete Algorithms, Philadelphia, PA, USA, 2009, pp. 596-605.

[6] C. Castellano, S. Fortunato, and V. Loreto, "Statistical Physics of Social Dynamics," APS Reviews of Modern Physics, vol. 81, no. 2, pp. 591646, 2009.

[7] M. Penrose and J. Yukich, "Central Limit Theorems For Some Graphs In Computational Geometry," Annals of Applied Probability, vol. 11, no. 4, pp. 1005-1041, 2001.

[8] _ _ "Weak Laws Of Large Numbers In Geometric Probability," Annals of Applied Probability, vol. 13, no. 1, pp. 277-303, 2003.

[9] D. Weitz, "Combinatorial Criteria for Uniqueness of Gibbs Measures," Random Structures \& Algorithms, vol. 27, no. 4, p. 445, 2005.

[10] P. Brémaud, Markov Chains: Gibbs fields, Monte Carlo simulation, and queues. Springer, 1999.

[11] G. Miller, S. Teng, W. Thurston, and S. Vavasis, "Separators for SpherePackings and Nearest Neighbor Graphs," J. of the ACM, vol. 44, no. 1, 1997.

[12] S. Winkler and S. Tatikonda, "Criteria for Rapid Mixing of Gibbs Samplers and Uniqueness of Gibbs Measures," in Allerton Conf. on Communication, Control, and Computing, 2006.

[13] M. Penrose, Random Geometric Graphs. Oxford University Press, 2003.

[14] J. Van Den Berg and C. Maes, "Disagreement Percolation in the Study of Markov Fields," The Annals of Probability, vol. 22, no. 2, pp. 749-763, 1994.

[15] H. Georgii, O. Haggstrom, and C. Maes., "The Random Geometry of Equilibrium Phases," in Phase Transitions and Critical Phenomena, C. Domb and J. Lebowitz, Eds. London: Academic Press, 2000, vol. 18, pp. $1-142$.

[16] Z. Kong and E. Yeh, "Percolation processes and wireless network resilience," in Information Theory and Applications Workshop, 2008, 2008, pp. 461-470. 